\title{
Can Lester Jones tubes be tolerated for decades?
}

\begin{abstract}
Purpose Lester Jones described canalicular bypass tubes 50 years ago. We present a cohort of patients with Jones' tubes first placed between 1969 and 1989, and who were reviewed within the last 15 years. Patients and methods Retrospective casenote review for living patients identified as having had Jones' tube placement prior to 1990. The duration of Jones' tube usage was noted and the number of replacements recorded.

Results Twenty-nine patients (33 eyes) had maintenance of their Jones' tube(s) within the last 15 years, and had first tube placement before 1990. The average follow-up was 29.5 years (median 28.8 years, range 17-45.7 years). The original tube was present in $8 / 33(24 \%)$ of eyes, at a mean survival of 34 years (33.3 years; range $29.4-44.4$ years). The number of tube replacements during follow-up ranged from 0 to 9 (mean 1.7; median 1). When considering the initially placed tube in all 33 eyes, however, the survival ranged between 18 days and 44.4 years (mean 13.6 years; median 6.9 years). At last follow-up, 11/33 (33\%) of eyes had lost

their tubes, with 9 having minimal or no symptoms.

Conclusions These patients with Jones' tube placement before 1990 provides the first recorded evidence that the device can be tolerated for at least four decades, and that some patients will-with appropriate outpatient maintenance-retain their originally placed tube. This information may be useful in counselling patients about the lifetime expectation for bypass tubes.

Eye (2018) 32, 142-145; doi:10.1038/eye.2017.168; published online 18 August 2017
\end{abstract}

Ophthalmology, Adnexal Service, Moorfields Eye Hospital NHS Foundation Trust, London, UK

\section{Correspondence:} GE Rose, Ophthalmology, Adnexal Service, Moorfields Eye Hospital NHS Foundation Trust, 162 City Road, London EC1V 2PD, UK

Tel: $+44(0) 2072533411$; Fax: +44(0)20 75662619 . E-mail: geoff.rose@ moorfields.nhs.uk

Received: 1 March 2017 Accepted in revised form: 22 May 2017

Published online:

18 August 2017

\section{Introduction}

Lester Jones described the Pyrex glass lacrimal canalicular bypass tube 50 years ago, ${ }^{1,2}$ following which many modifications have been
RL Scawn, DH Verity and GE Rose

described in an attempt to reduce spontaneous loss including a central external bulge in the tube, porous polythene coating, external frosting of the glass, silicone tips, and more recently, an intranasal silicone flange. ${ }^{3-7}$ However, because of its low surface area and surface reactivity, we consider the original Jones' tube to remain the 'gold-standard' for treating disabling epiphora due to canalicular failure.

Patients with canalicular agenesis, or those with acquired canalicular block due to primary Herpetic conjunctivitis or trauma, are generally young and often present in their third or fourth decades. ${ }^{8}$ Although Jones originally suggested that epithelisation of the tract may allow the tube to be dispensed with, studies have not found this to be common, ${ }^{9}$ and the prosthesis should generally be considered as being required for perpetuity. ${ }^{9,10}$ This study looks specifically at a small cohort of patients who have had the opportunity for at least 25 years of Jones' tube usage, to assess tolerance for placement of this device over several decades.

\section{Patients and methods}

Three hundred and ten identifiable patients from a previous study ${ }^{9}$ had initial implantation of Jones' tubes between 1969 and 1989, with surgical techniques described in the original paper. $^{9}$

Electronic and paper records were reviewed where available, and the study cohort restricted to those with follow-up data to at least the year 2000-giving a potential follow-up of at least one decade (potentially between 11 and 46 years). For patients meeting these inclusion criteria, the total number of tube replacements was recorded-such replacements being for spontaneous loss or tube repositioning. Excision of redundant conjunctiva or granulation tissue was not regarded as an 'event', unless the tube was repositioned or a new tube inserted. 


\section{Results}

Twenty-nine patients $(29 / 310 ; 9.4 \%)$ met the inclusion criteria-with 33 eyes having Jones' tubes-and their period from original placement to latest follow-up ranged from 17 to 45.7 years (mean 29.5 years, median 28.8 years).

The aetiology for canalicular block was known in 97\% $(32 / 33)$ cases, the commonest being post-Herpetic obstruction (24\%), congenital causes (18\%), post-radiation $(12 \%)$, and failed multiple lacrimal surgery $(12 \%)$

(Table 1).

A Jones' tube was in situ in 22/33 (67\%) eyes at last clinic review and these patients had good control of epiphora. Eleven patients did not have a tube in place at their last visit, and nine (81\%) had only mild or no epiphora and did not request replacement of the device; these nine patients were therefore discharged from ophthalmic care. Of the two with residual symptoms at the last clinic visit, one patient failed to attend for tube replacement (presumably finding symptoms acceptable without the device), and tube replacement was discouraged in the other patient, who had mild exposure keratopathy due to facial palsy.

In 32 eyes where survival of the first placed tube was known, it varied widely from just 18 days to 44.4 years, with an average survival of 13.6 years (median 6.9 years) -and retention of the initially placed device in thisalbeit selected-cohort was just under $50 \%$ at 5 years, 30\% at 20 years, and $25 \%$ beyond 30 years (Figure 1). Eight eyes (24\%) still had the original device in place with $6 / 8$ (75\%) placed in the 1970s and 2/8 (25\%) in the 1980s. Survival of the initial tube was notably longer for congenital atresia (mean 16.1 years) and post-herpetic block (mean 16.9 years), as compared to that in patients with facial palsy ( $<3$ months) or after trauma (11 months).

Spontaneous loss of the tube (in 15/21 cases; 71\%) was the commonest reason for replacement of the initial device, but other reasons included three cases with replacement of a buried tube (14\%), and three cases with malposition. The total number of tube replacements, over a follow-up of between 17 and 45.7 years, ranged from 0 to 9 (mean 1.7 replacements). Other procedures included excision of minor conjunctival granulomas or inclusion cysts in five patients, or reduction of conjunctivochalasis (1 patient) and open revision of the osteotomy (1 patient). One patient later elected to have partial dacryoadenectomy for gustatory lacrimation.

\section{Discussion}

With availability of long-term records for some patients with Jones' tube placement at least 25 years ago, this

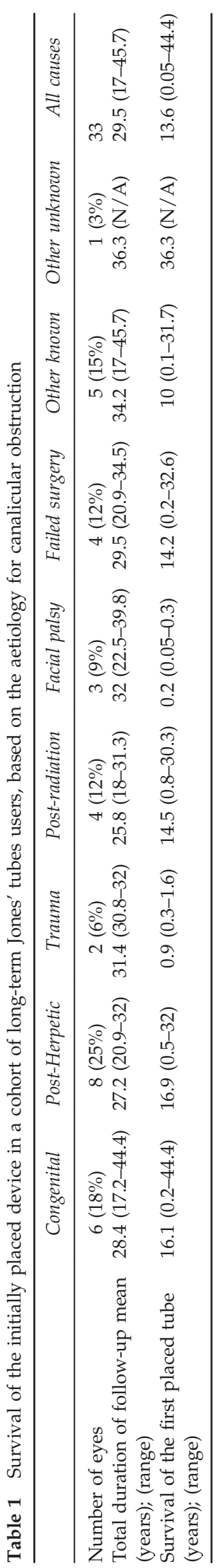




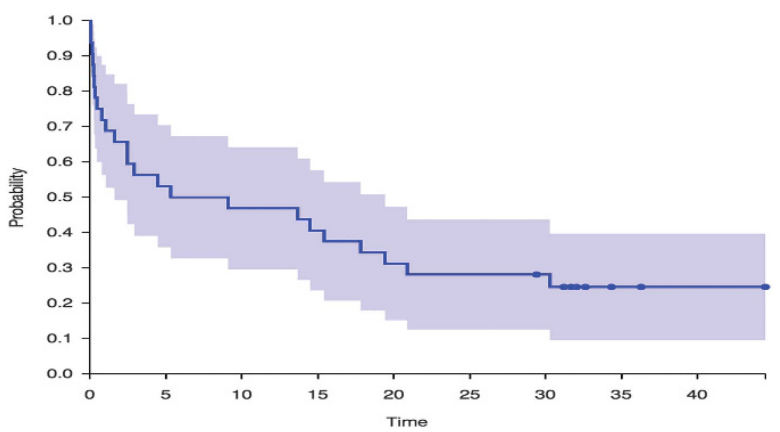

Figure 1 The probability of the device being still in situ at a given time (in years) after initial placement of Jones' tubes in 32 eyes prior to 1990 (Kaplan-Meier analysis). Shaded area represents $95 \%$ confidence interval.

retrospective study provides a unique insight into whether glass canalicular bypass tubes are tolerated over decades of placement. Jones' tubes are often portrayed as being associated with complications, including tube displacement and patient dissatisfaction. ${ }^{11,12}$ Tube displacement is most frequent in the early months after placement, with Sekhar reporting two-thirds occurring in the first 3 months, ${ }^{11}$ and we find a similar predilectionalthough with only $15 \%$ tubes failing within 3 months. Notably, for our patients with very long-term success, attrition rates appear to drop in the third year, that $50 \%$ of original tubes are still in situ at 5 years, and that there are no further tube losses beyond 30 years (Figure 1).

Eleven eyes had no tube in situ at the last clinic review, with more than $80 \%$ of these eyes being asymptomatic or reporting minimal symptoms. Jones originally postulated that, due to epithelisation of the tract with time, the device might not be necessary, but multiple studies have shown that only $3-7 \%$ of patients achieve symptom control without a tube. ${ }^{9,11,13}$ The $27 \%(9 / 33)$ asymptomatic tubeless patients in this study suggests that drainage might become established in a higher proportion of cases -but possibly only after decades of tube placement? It should be noted, however, that the sub-group of nine asymptomatic eyes without tubes included all of the eyes with post-radiation occlusion (4 cases) and StevensJohnson syndrome (2 eyes) — this suggesting that a reduced tear production due to disease might have helped obviate the need for a drainage conduit. ${ }^{14}$

With the exception of one patient after trachoma, the cohort of eight patients who retained their original tube (for an average of 34 years) all had conditions that did not impair ocular surface lubrication-possibly suggesting that a normal conjunctival surface might aid long-term Jones' tube survival. Despite this, however, it is notable that the four post-radiation patients-who had all lost their initial tube — still had an average tube usage of 14 years.

The retrospective design for this study has limitations, the main one being death of patients over the intervening 25 years-but we only intended to examine whether usage of such devices could be tolerated for several decades. Whilst inclusion of patients with follow-up within the last 15 years might have biased the series to optimal outcomes, this bias will have been counteracted both by the return of patients to their referring institutions after successful tube placement (Moorfields Eye Hospital being a national tertiary referral centre) and by a known failure of patients to attend for follow-up tube cleaning when the device is working well. However, despite its limitations, this investigation provides reassuring evidence that the original Jones' Pyrex glass canalicular bypass tube can be tolerated-and control symptomsfor several decades.

\section{Summary}

What was known before

- Lester Jones tubes are indicted for disabling epiphora secondary to canalicular failure. Lester Jones tube are associated with complications including extrusion and spontaneous loss.

What this study adds

- The longest published follow-up on Lester Jones tubes. Demonstrates successful tube retention, spanning more than four decades, can be achieved in some patients.

\section{Conflict of interest}

The authors declare no conflict of interest.

\section{References}

1 Jones LT. Conjunctivodacryocystorhinostomy. Am J Ophthalmol 1965; 59: 773-783.

2 Jones LT. An anatomical approach to problems of the eyelids and lacrimal apparatus. Arch Ophthalmol 1961; 66: 111-124.

3 Fan X, Bi X, Fu Y, Zhou H. The use of Medpor coated tear drainage tube in conjunctivodacryocystorhinostomy. Eye 2008; 22: 1148-1153.

4 Dailey RA, Tower RN. Frosted Jones Pyrex tubes. Ophthal Plast Reconstr Surg 2005; 21: 185-187.

5 Gladstone GJ, Putterman AM. A modified glass tube for conjunctivo-dacryocystorhinostomy. Arch Ophthalmol 1985; 103: 1229-1230.

6 Hung YJ, Kim G, Sohn BK. Conjunctivorhinostomy with rubber-tipped Jones tube. Ann Plast Surg 2004; 52: 68-71.

7 Bagdonaite L, Pearson AR. Early experience with the StopLoss Jones tube. Orbit 2015; 34: 132-136.

8 McLean CJ, Rose GE. Postherpetic lacrimal obstruction. Ophthalmology 2000; 107: 496-499. 
9 Rose GE, Welham RA. Jones' lacrimal canalicular bypass tubes: twenty-five years' experience. Eye 1991; 5: 13-19.

10 Lim C, Martin P, Benger R, Kourt G, Ghabrial R. Lacrimal canalicular bypass surgery with the Lester Jones tube. Am J Ophthalmol 2004; 137: 101-108.

11 Sekhar GC, Dortzbach RK, Gonnering RS, Lemke BN. Problems associated with conjunctivodacryocystorhinostomy. Am J Ophthalmol 1991; 112: 502-506.
12 Rosen N, Ashkenazi I, Rosner M. Patient dissatisfaction after functionally successful conjunctivodacryocystorhinostomy with Jones tube. Am J Ophthalmol 1994; 117: 636-642.

13 Zilelioğlu G, Gündüz K. Conjunctivodacryocystorhinostomy with Jones tube. A 10-year study. Doc Ophthalmol 1996-1997; 92: 97-105.

14 Ozdemir M, Temizdemir H. Age- and gender-related tear function changes in normal population. Eye 2010; 24: 79-83. 